Canad. Math. Bull. Vol. 19 (1), 1976

\title{
PSEUDO-NOETHERIAN RINGS
}

BY

KENNETH P. MCDOWELL

1. Introduction. In the latter part of the 1950's some interesting papers appeared (e.g. [2] and [10]) which examined the relationships occurring between the purely algebraic and homological aspects of the theory of finitely generated modules over Noetherian rings. Many of these relationships remain valid if one considers the much wider class of rings determined by the following definition.

Definition. A commutative ring $R$ is called pseudo-Noetherian if it satisfies the following two conditions.

(1) $R$ is coherent.

(2) If $M$ is a non-zero finitely presented $R$-module and $I$ is a finitely generated ideal of $R$ contained in the zero-divisors of $M$, then there exists a non-zero $m$ in $M$ with $I m=0$.

A fundamental link between algebraic and homological concepts is established in the main result of Section 2.

THEOREM. Let $R$ be a local pseudo-Noetherian ring and $M$ a non-zero finitely presented $R$-module. Then the length of any maximal $M$-sequence is equal to the depth of $M$ and if $M=R$, this value is the finistic dimension of $R$.

Section 3 introduces a method for constructing new pseudo-Noetherian rings.

THEOREM. A faithfully flat directed colimit of pseudo-Noetherian domains is pseudo-Noetherian.

The usefulness of this result is evident in Section 4 which presents various examples of (non-Noetherian) pseudo-Noetherian rings. Section 4 also provides an example of a coherent local ring which is not pseudo-Noetherian.

2. Pseudo-Noetherian Rings. All rings will be commutative with 1 and all modules and ring homomorphisms unitary. $\mathbf{N}$ will denote the natural numbers and $\mathbf{Z}$ the integers. A local ring is a (not necessarily Noetherian) ring $R$ with a unique maximal ideal $\mathrm{m}$ and such a ring will be denoted by $(R, \mathrm{~m})$. A ring $R$ is called coherent if each of its finitely generated ideals is finitely presented. (See [4] and [6] for a detailed discussion of coherent rings). For a module $M, p \cdot \operatorname{dim}_{R} M$ ( $p \cdot \operatorname{dim} M$ if no confusion arises) will denote the projective dimension of $M$. The

Received by the editors December 23, 1974. 
weak global dimension of $R$ will be denoted by $w \cdot \mathrm{gl} \cdot \operatorname{dim} R$ For a coherent $\operatorname{ring} R$,

$w \cdot \mathrm{gl} \cdot \operatorname{dim} R=\sup \{p \cdot \operatorname{dim} M \mid M$ a finitely presented $R$-module $\}$. For such a ring, define the finistic dimension $(f \cdot \operatorname{dim} R)$ as follows.

$f \cdot \operatorname{dim} R=\sup \{p \cdot \operatorname{dim} M \mid M$ a finitely presented $R$-module of finite projective dimension\}.

For a module $M$ over a ring $R, Z(M)$ will denote the set of all elements of $R$ which are annihilated by a non-zero element of $M$. A (possibly infinite) sequence of elements $x_{1}, x_{2}, \ldots$ of the ring $R$ will be called an $M$-sequence if $\left(\sum_{i} x_{i} R\right) M \neq M$ and for each $i \geq 1, x_{i} \notin Z\left(M /\left(\sum_{j=1}^{i-1} x_{j} R\right) M\right)$. The length of a finite $M$-sequence is defined to be the cardinality of the set of elements of the sequence. A sequence which is not finite is said to have length infinity. An $M$-sequence is termed maximal if it is not an initial subsequence of some other $M$-sequence. In this sense maximal $M$-sequences for finitely generated $M$ always exist.

Definition 2.1. ([1]) Let $R$ be a ring and $M$ and $N$ two $R$-modules. If there exists an integer $m$ such that $\operatorname{Ext}_{R}^{m}(M, N) \neq 0$ define

$$
M \text {-depth } N=\inf \left\{n \mid \operatorname{Ext}_{R}^{n}(M, N) \neq 0\right\} .
$$

Otherwise, $M$-depth $N=\infty$.

The following lemma indicates the relationship between this type of depth and the concept of an $M$-sequence.

LEMMA 2.2. Let $R$ be a pseudo-Noetherian ring, I a finitely generated ideal and $M$ a finitely presented $R$-module with $I M \neq M$. Then the length of any $M$-sequence maximal in $I$ is equal to $(R / I)$-depth $M$.

Proof. Let $x_{1}, \ldots, x_{n}$ be an $M$-sequence in $I$. Because $M /\left(x_{1}, \ldots, x_{n}\right) M$ is a finitely presented module over the pseudo-Noetherian ring $R, x_{1}, \ldots, x_{n}$ is maximal in $I$ if and only if $\operatorname{Hom}_{R}\left(R / I, M /\left(x_{1}, \ldots, x_{n}\right) M\right) \neq 0$. Appendix 3-1 of [8] indicates that this is equivalent to $\operatorname{Ext}_{R}^{n}(R / I, M) \neq 0$. Since this last condition refers only to the length of the $M$-sequence being considered, it is clear that all $M$-sequences maximal in $I$ have the same length. If $x_{1}, \ldots, x_{n}$ is such a sequence, the above considerations show that $(R / I)$-depth $M=n$. Similarly, if there exists an infinite $M$-sequence in $I \operatorname{Ext}_{R}^{m}(R / I, M)=0$ for all $m \geq 0$ and hence, $(R / I)$ depth $M=\infty$.

It should perhaps be noted here that a local coherent ring $R$ is pseudo-Noetherian if and only if the statement of the above lemma is valid. The lemma indicates that, if $R$ is a local pseudo-Noetherian ring and $I$ and $J$ are two finitely generated proper ideals with $I \subseteq J$, then $(R / I)$-depth $M$ does not exceed $(R / J)$-depth $M$ for any finitely presented module $M$.

Definition 2.3. For a local pseudo-Noetherian ring $(R, \mathfrak{m})$ and a non-zero 
finitely presented module $M, \sup \{(R / I)$-depth $M \mid I$ a proper finitely generated ideal $\}$ will be denoted $\operatorname{depth}_{R} M$ (depth $M$ if no confusion arises). Notice that if $(R, \mathfrak{m})$ is Noetherian, depth $M=(R / \mathfrak{m})$-depth $M$.

Lemma 2.2 may now be generalized.

THEOREM 2.4. Let $(R, \mathfrak{m})$ be a local pseudo-Noetherian ring and $M$ a non-zero finitely presented $R$-module. Then the lengths of all maximal $M$-sequences are the same and equal to depth $M$.

Proof. Suppose depth $M=n<\infty$ and let $s$ be a maximal $M$-sequence. Denote the length of $s$ by $\ell(s)$. Assume $\ell(s)>n$ and let $I_{n+1}$ denote the ideal generated by the first $n+1$ elements of $s$. $\left(R / I_{n+1}\right)$-depth $M \leq$ depth $M=n$ and, hence, by Lemma 2.2, $I_{n+1}$ cannot contain an $M$-sequence of length $n+1$. This contradiction shows that $\ell(s) \leq$ depth $M$. Now assume $\ell(s)=m<n$ and let $J$ be a proper finitely generated ideal containing $s$ for which $(R / J)$-depth $M=n$. By Lemma 2.2 there exists an $M$-sequence maximal in $J$ of length $n$. Since $J$ cannot contain two maximal $M$-sequences of different length, the assumption was incorrect and $\ell(s)=\operatorname{depth} M$. If depth $M=\infty$ similar techniques show that all maximal $M$-sequences have infinite length.

If $R$ is a pseudo-Noetherian ring and $I$ is a finitely generated ideal then $R / I$ is also pseudo-Noetherian. Suppose $(R, m)$ is a local pseudo-Noetherian ring and $M$ is a non-zero finitely presented module. Furthermore, assume there exists $x$ in $\mathrm{m}$ with $x \notin Z(M)$. Theorem 2.4 may be used to show that $\operatorname{depth}_{R} *(M / x M)=$ $\operatorname{depth}_{R} M-1$ where $R^{*}=R /(x)$. If, moreover, $x \notin Z(R), p \cdot \operatorname{dim}_{R} M=p \cdot \operatorname{dim}_{R^{*}}(M /$ $x M)$. (This is the Third Change of Rings Theorem for coherent rings.) These observations are employed in the next theorem.

THeORem 2.5. Let $(R, \mathrm{~m})$ be a local pseudo-Noetherian ring. The following three quantities are equal.

(1) the length of any maximal $R$-sequence.

(2) $f \cdot \operatorname{dim} R$.

(3) depth $R$.

Proof. For any local coherent ring $R$ and non-zero-divisor $x$ in the radical, $f \cdot \operatorname{dim} R^{*}=f \cdot \operatorname{dim} R-1 \quad\left(R^{*}=R /(x)\right)$. Moreover, $f \cdot \operatorname{dim} R=0$ if and only if every proper finitely generated ideal has a non-zero annihilator (cf. [3], Corollary 5.6). If $s$ is a maximal $R$-sequence, these facts may be used to show that $\ell(s) \leq$ $f \cdot \operatorname{dim} R$ be means of induction on $f \cdot \operatorname{dim} R$.

Induction on depth $R$ will now be employed to demonstrate that $f \cdot \operatorname{dim} R \leq$ depth $R$. If depth $R=0, f \cdot \operatorname{dim} R=0$ by the above characterization of zero finitistic dimension. Now assume depth $R=n>0$ and let $M$ be any finitely presented module with finite projective dimension $m \geq 1$. Let $0 \rightarrow K \rightarrow F \rightarrow M \rightarrow 0$ be a finite presentation of $M$ ( $F$ finitely generated free, $K$ finitely generated). Since depth $R>0$ there 
exists a non-zero-divisor $x$ in $\mathfrak{m}$. Hence $x \notin Z(K)$. Now $K$ is finitely presented and the induction assumption may be employed to obtain $m-1=p \cdot \operatorname{dim}_{R} K=$ $p \cdot \operatorname{dim}_{R} *(K / x K) \leq f \cdot \operatorname{dim} R^{*} \leq \operatorname{depth}_{R^{*}} R^{*}=n-1$ where, as usual, $R^{*}=R /(x)$. This shows that $p \cdot \operatorname{dim} M=m \leq n=\operatorname{depth} R$ and, therefore, that $f \cdot \operatorname{dim} R \leq$ depth $R$.

Thus, if $s$ is a maximal $R$-sequence, $\ell(s) \leq f \cdot \operatorname{dim} R \leq$ depth $R$ and Theorem 2.4 shows that the above three quantities are in fact equal.

REMARK. Associated prime ideals play an important role in the original treatment of the above material for Noetherian rings. Although there is a theory of associated primes which may be employed here in a somewhat analogous manner, reference to prime ideals is not actually needed in the pseudo-Noetherian situation.

3. New Pseudo-Noetherian Rings from Old. In this section it will be demonstrated that rings which occur as a particular type of union of other pseudo-Noetherian rings (e.g. Noetherian integral domains) are themselves pseudo-Noetherian.

A ring homomorphism $\psi: A \rightarrow R$ between two rings $A$ and $R$ is called faithfully flat if $R$ is a faithfully flat $A$-module via $\psi$. Such maps are always 1-1. Moreover, $\psi$ is faithfully flat if and only if $R$ is a flat $A$-module and $A$ is a pure $A$-submodule of $R$. (These and other results concerning faithful flatness may be found in [4].)

Definition 3.1. Let $D$ be an updirected partially ordered set and $S=\left(R_{d}, f_{d d^{\prime}}\right)$ a corresponding directed system of rings and ring homomorphisms. $S$ is called a faithfully flat directed system if for each $\left(d, d^{\prime}\right) \in D \times D$ with $d \leq d^{\prime}, f_{d d^{\prime}}$ is faithfully flat. In this case, the colimit of $S$ is called a faithfully flat directed colimit of the family of rings $\left(R_{d}\right)_{d \in D}$.

LEMMA 3.2. Let $A$ be a subring of a ring $R$. Let $M$ be a finitely presented $R$-module with finite presentation

$$
0 \longrightarrow K \longrightarrow R^{n} \stackrel{\beta}{\longrightarrow} M \longrightarrow 0
$$

such that the finitely many generators $k_{1}, \ldots, k_{m}$ of $K$ lie n $A^{n}$. Let $K^{\prime}=\sum_{i=1}^{m} A k_{i}$ and consider

$$
0 \longrightarrow K^{\prime} \longrightarrow A^{n} \stackrel{\beta^{\prime}}{\longrightarrow} M^{\prime} \longrightarrow 0 \quad\left(M^{\prime}=\operatorname{coker}\left(K^{\prime} \longrightarrow A^{n}\right)\right) .
$$

Then, $M^{\prime} \otimes_{A} R \cong M$.

Proof.

$$
\begin{array}{ll}
R^{m} \stackrel{\alpha}{\longrightarrow} R^{n} \stackrel{\beta}{\longrightarrow} M \longrightarrow 0 \text { is exact. } & \alpha\left(r_{1}, \ldots, r_{m}\right)=\sum_{i=1}^{m} r_{i} k_{i} . \\
A^{m} \stackrel{\alpha^{\prime}}{\longrightarrow} A^{n} \stackrel{\beta^{\prime}}{\longrightarrow} M^{\prime} \longrightarrow 0 \text { is exact. } & \alpha^{\prime}\left(a_{1}, \ldots, a_{m}\right)=\sum_{i=1}^{m} a_{i} k_{i} .
\end{array}
$$

Define $A^{m} \underset{A}{\otimes} \stackrel{h}{\longrightarrow} R^{m}$ by $h\left(\left(a_{1}, \ldots, a_{m}\right) \underset{A}{\otimes} r\right)=\left(a_{1} r, \ldots, a_{m} r\right)$. 
Define $A^{n} \underset{A}{\otimes} \stackrel{\mathrm{g}}{\longrightarrow} R^{n}$ by $g\left(\left(a_{1}, \ldots, a_{n}\right) \underset{A}{\otimes} r\right)=\left(a_{1} r, \ldots, a_{n} r\right)$.

$h$ and $\mathrm{g}$ are isomorphisms and the following diagram commutes with exact rows.

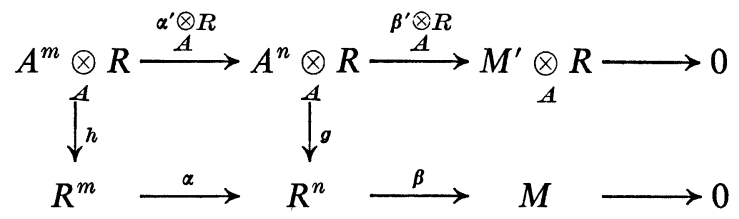

The induced map between the cokernels which makes the second square commute is clearly onto and straightforward diagram chasing shows it also to be injective. This map is the required isomorphism.

THEOREM 3.3. If a ring $R$ is a faithfully flat directed colimit of pseudo-Noetherian integral domains, then $R$ is pseudo-Noetherian.

Proof. First observe that $R$ is coherent ([11], Proposition 20) and that $R$ is actually a directed union of pseudo-Noetherian rings since the maps involved in the directed system are monomorphisms. Therefore, let $R=\bigcup_{\beta \in B} R_{\beta}$ where $B$ is an infinite set, $R_{\beta}$ is a pseudo-Noetherian domain for each $\beta \in B$, and the union is directed. Now let $I$ be a finitely generated ideal of $R$ and $M$ a non-zero finitely presented $R$-module with $I \subseteq Z(M)$. The goal of this proof is to exhibit a non-zero $m$ in $M$ with $I m=0$.

Suppose $0 \rightarrow K \rightarrow R^{s} \rightarrow M \rightarrow 0$ is a finite presentation for $M$. Without loss of generality, assume that $K$ is a submodule of $R^{s}$ generated by $\left\{k_{i} \mid 1 \leq i \leq t\right\}$ where $k_{i}=\left(k_{i 1}, \ldots, k_{i s}\right)$. Moreover, suppose $I$ is generated by $\left\{a_{\ell} \mid 1 \leq \ell \leq u\right\}$. $(s, t$, and $u$ are natural numbers). Now choose $\beta_{0} \in B$ such that $\left\{k_{i j} \mid 1 \leq i \leq t, 1 \leq j \leq s\right\} \bigcup$ $\left\{a_{\ell} \mid 1 \leq \ell \leq u\right\} \subseteq R_{\beta_{0}}$. Let $I^{\prime}=\sum_{\ell=1}^{u} R_{\beta_{0}} a_{\ell}, K^{\prime}=\sum_{i=1}^{t} R_{\beta_{0}} k_{i}$ and suppose $M^{\prime}$ is given by the short exact sequence $0 \rightarrow K^{\prime} \rightarrow R_{\beta_{0}}^{s} \rightarrow M^{\prime} \rightarrow 0$. Then $I^{\prime}$ is a finitely generated ideal of $R_{\beta_{0}}$ and $M^{\prime}$ is a non-zero finitely presented $R_{\beta_{0}}$-module with $M^{\prime} \otimes_{R_{\beta_{0}}} \cong \cong$ $M$ (Lemma 3.2).

It will now be shown that $I^{\prime} \subseteq Z\left(M^{\prime}\right)$. Let $x \in I^{\prime} \subseteq I$. Since $I \subseteq Z(M)$, there exists a non-zero $m$ in $M$ with $x m=0$. Assume that $x$ has zero annihilator in $M^{\prime}$. Exercise 5, p. 122 of [5], shows that $M^{\prime} \otimes_{R_{\beta_{0}}} x R_{\beta_{0}} \rightarrow M^{\prime}$ is 1-1 and therefore

$$
M^{\prime} \underset{R_{\beta_{0}}}{\otimes} x R_{\beta_{0}} \underset{R_{\beta_{0}}}{\otimes} R \rightarrow M^{\prime} \underset{R_{\beta_{0}}}{\otimes} R
$$

is 1-1. Now since $M^{\prime} \otimes_{R_{\beta_{0}}} R \cong M$ and

$$
x R_{\beta_{0}} \underset{R_{\beta_{0}}}{\otimes} R \cong x R, \quad M^{\prime} \underset{R_{\beta_{0}}}{\otimes} x R \rightarrow M
$$

is 1-1. But again because $M^{\prime} \otimes_{R_{\beta_{0}}} R \cong M$, this shows that $M \otimes_{R} x R \rightarrow M$ is $1-1$. Hence $x R^{s} \cap K=x K$ ([5], Exercise 5, p. 122) which demonstrates that $m=0$ since $x m=0$ and $x \notin Z(R)$. This is a contradiction. Therefore, the original assumption is false and $I^{\prime} \subseteq Z\left(M^{\prime}\right)$ as required. 
Since $R_{\beta_{0}}$ is pseudo-Noetherian, there exists a non-zero $m^{\prime}$ in $M^{\prime}$ with $I^{\prime} m^{\prime}=0$. Because $R$ is a directed union of rings faithfully flat over $R_{\beta_{0}}, R$ is faithfully flat over $R_{\beta_{0}}$. Hence $R_{\beta_{0}}$ is a pure submodule of $R$ and therefore the middle map in the following sequence is injective.

$$
M^{\prime} \cong M^{\prime} \underset{R_{\beta_{0}}}{\otimes} R_{\beta_{0}} \rightarrow M^{\prime} \underset{R_{\beta_{0}}}{\otimes} R \cong M
$$

Since $m^{\prime}$ can be thought of as lying in $M$ and since $I^{\prime}$ and $I$ are generated by the same elements (albeit over different rings), $I^{\prime} m^{\prime}=0$ implies $I m^{\prime}=0$ completing the proof.

4. Examples. Every coherent Bézout ring is pseudo-Noetherian because all of its finitely generated ideals are principal. Hence, besides Noetherian rings, the class of pseudo-Noetherian rings contains all Bézout domains (e.g. valuation domains), coherent generalized valuation rings, and von Neumann regular rings. Moreover, any coherent ring which is a zero-divisor ring in the sense of E. G. Evans ([7]) is pseudo-Noetherian. Theorem 3.3 will now be used to construct further examples.

EXAMPLE 1. Let $A$ be a noetherian domain and $\left\{x_{n} \mid n \in N\right\}$ an infinite set of indeterminates. Denote by $R$ the subring of $A\left[\left[\left(x_{n}\right)_{n \in N}\right]\right]$ consisting of all those elements whose expansions contain only finitely many indeterminates. Then $R$ is pseudo-Noetherian.

In fact, for any finite subset $F$ of $N$ let $R_{F}=A\left[\left[\left(x_{n}\right)_{n \in F}\right]\right]$. Each such $R_{F}$ is Noetherian (hence pseudo-Noetherian) and $R$ is a faithfully flat directed union of $\left\{R_{F^{\prime}} \mid F\right.$ a finite subset of $\left.N\right\}$. (If $F \subseteq F^{\prime}, R_{F}$, is isomorphic as $R_{F^{\prime}}$-module to a direct product of copies of $R_{F^{\prime}}$. Hence, since $R_{F}$ is Noetherian, $R_{F^{\prime}}$ is a flat $R_{F^{-}}$ module ([6]). Since the inclusion $R_{F} \rightarrow R_{F}$, is local, this is enough to show faithful flatness). Thus, by Theorem 3.3, $R$ is pseudo-Noetherian. Of course if $A$ is a local Noetherian domain -in particular a field $-R$ is a local pseudo-Noetherian ring. (The above construction may be imitated by considering polynomials instead of power series.)

EXAmple 2. The first example has infinite weak global dimension. For any positive integer $n$, a local pseudo-Noetherian ring of weak global dimension $n$ may be constructed as follows. Let $K$ be a field and $\left\{x_{i j} \mid 1 \leq i \leq n, j \in \mathbf{N}\right\}$ an infinite set of indeterminates. For each $m \in \mathbf{N}$ define $R_{m}=K\left[\left[x_{1 m}, \ldots, x_{n m}\right]\right]$ and a map $f_{m}^{m+1}: R_{m} \rightarrow R_{m+1}$ given by $f_{m}^{m+1}\left(x_{i m}\right)=x_{i m+1}^{2}(1 \leq i \leq n) . R_{m}$ is of course Noetherian and $f_{m}^{m+1}$ is an injective ring homomorphism. Let $R$ be the colimit of the corresponding directed system of rings and ring homomorphisms.

$R$ is pseudo-Noetherian. In fact, observe that if $m$ and $m^{\prime}$ are natural numbers and $m \leq m^{\prime}, R_{m^{\prime}}$ is a finitely generated free $R_{m}$-module. Indeed, $\left\{x_{1 m^{\prime}}^{s_{1}}, x_{2 m^{\prime}}^{s_{2}} \ldots\right.$ $\left.x_{n m^{\prime}}^{s_{n}} \mid 0 \leq s_{i}<2^{m^{\prime}-m}(1 \leq i \leq n)\right\}$ is a free $R_{m}$-basis for $R_{m^{\prime}}$. Hence $R_{m^{\prime}}$ is a flat $R_{m}$-module and $R_{m}$ is pure in $R_{m^{\prime}}$. By Theorem 3.3, $R$ is pseudo-Noetherian. 
$R$ may be described as that subring of the power series ring

$$
K\left[\left[x_{1}, x_{1}^{1 / 2}, x_{1}^{(1 / 2)^{2}}, \ldots x_{2}, x_{2}^{1 / 2}, \ldots x_{n}, x_{n}^{1 / 2}, \ldots\right]\right]
$$

consisting of all elements which do not contain roots of arbitrarily high degree. Hence, it is clear that $R$ is non-Noetherian for $\left(x_{1}\right) \subset\left(x_{1}^{1 / 2}\right) \subset\left(x_{1}^{(1 / 2) 2}\right) \subset \cdots$ is an infinite properly ascending chain of ideals. By a result of Osofsky gl $\cdot \operatorname{dim} R \leq$ $n+1<\infty$ ([9] Proposition 2.1) and since the weak global dimension of $R$ is therefore finite, $f \cdot \operatorname{dim} R=w \cdot \mathrm{gl} \cdot \operatorname{dim} R$. By Theorem $2.5 w \cdot \mathrm{gl} \cdot \operatorname{dim} R=n$ because $x_{1}, \ldots, x_{n}$ is a maximal $R$-sequence.

Theorem 3.3 and techniques similar to those used above may also be employed to demonstrate that if $R$ is a Noetherian domain and $G$ is a torsion free abelian group, then the group ring $R G$ is pseudo-Noetherian. The final example demonstrates that, in general, coherent local rings need not be pseudo-Noetherian.

Example 3. In [12] V. W. Vasconcelos shows that every local ring of global dimension two is a coherent integral domain. It is furthermore proven that if the maximal ideal is principal or not finitely generated, the ring is a valuation domain. Otherwise, the maximal ideal is generated by two elements, and the non-finitely generated prime ideals are flat, countably generated, linearly ordered, and contained in any finitely generated prime ideal.

Consider the following example of such a ring. Let $A$ represent the polynomial ring $\mathbf{Z}[x]$ localized at the ideal $(2, x)$ and let $K$ denote the quotient field of this Noetherian local ring of global dimension two. Now let $R$ be that subring of the power series ring $K[[t]]$ consisting of all elements with constant term lying in $A$. In [12] this ring is shown to be a local ring of global dimension two which is neither a valuation domain nor a Noetherian ring.

Let $\mathfrak{m}$ be the maximal ideal of $A$ and $\mathfrak{M}$ the maximal ideal of $R$ consisting of all those elements of $R$ with constant term lying in $\mathrm{m}$. It will first be shown that $\mathfrak{M}=Z(R / t R)$. In fact, suppose $r$ is any member of $\mathfrak{M}$ with constant $m \in \mathfrak{m}$. Without loss of generality $m$ may be considered non-zero since $t K R \subseteq Z(R / t R)$. Therefore, let $r^{\prime}=(1 / m) t$. Since $m \in \mathfrak{m},(1 / m) \notin A$ and $r^{\prime} \notin t R$. However, $r r^{\prime}$ is obviously a member of $t R$ showing that $r \in Z(R / t R)$. Hence, $\mathfrak{M}=Z(R / t R)$.

Now by previous remarks $\mathfrak{M}$ is finitely generated (by two elements) and therefore, to show $R$ is not pseudo-Noetherian it is only necessary to demonstrate that no non-zero element of the cyclically presented module $R / t R$ annihilates $\mathfrak{M}$. That is, we must show that if $r \in R$ with $r \mathfrak{M} \subseteq t R$, then $r \in t R$. Such an $r$ must have zero constant term since by hypothesis $r m \in t R$ for any non-zero $m$ in $\mathrm{m}$. If $k=(a / b)$ $(a, b \in A)$ is the coefficient of $t$ in $r, b \mid a m$ for all $m$ in $m$ for the same reason. But since $A$ is a unique factorization domain, $b$ may be written as a finite product of powers of prime elements and with this it is easy to see that $b$ must divide $a$ and hence $k \in A$ showing that $r \in t R$ as required. Hence, $R$ is not a pseudo-Noetherian ring. 


\section{REFERENCES}

1. M. Auslander and M. Bridger, Stable module theory, Mem. Amer. Math. Soc. 94 (1969).

2. M. Auslander and D. Buchsbaum, Homological dimension in local rings, Trans. Amer. Math. Soc. 85 (1957), 390-405.

3. H. Bass, Finitistic dimension and a homological generalization of semi-primary rings, Trans. Amer. Math. Soc. 95 (1960), 466-488.

4. N. Bourbaki, Éléments de Mathématique XXVII Algèbre Commutative, Hermann, Paris, 1961.

5. H. Cartan and S. Eilenberg, Homological Algebra, Princeton University Press, Princeton, 1956.

6. S. U. Chase, Direct products of modules, Trans. Amer. Math. Soc. 97 (1960), 457-473.

7. E. G. Evans, Jr., Zero divisors in Noetherian-like rings, Trans. Amer. Math. Soc. 155 (1971), 505-512.

8. I. Kaplansky, Commutative Rings, Allyn and Bacon, Boston, 1970.

9. B. L. Osofsky, Upper bounds on homological dimension, Nagoya Math. J. 32 (1968), 315-322.

10. D. Rees, The grade of an ideal or module, Proc. Cambridge Philos. Soc. 53 (1957), 28-42.

11. J. P. Soublin, Anneaux et modules cohérents, J. Algebra 15 (1970), 455-472.

12. W. V. Vasconcelos, The local rings of global dimension two, Proc. Amer. Math. Soc. 35 (1972), 381-386.

Department of Mathematics

MCMASTER UNIVERSITY

HAMILTON, ONTARIO

Canada L8S 4K1

Present address:

Department of Mathematics

WILFRID LAURIER UNIVERSITY

WATERLOO, Ontario

CANADA N2L 3C5 